\title{
Editorial
}

\section{On the Right Track: Implementing Interventions to Reduce Readmissions in Dialysis Patients}

\author{
Laura C. Plantinga ${ }^{a} \quad$ Bernard G. Jaar ${ }^{b}$ \\ ${ }^{a}$ Departments of Medicine and Epidemiology, Emory University, Atlanta, GA, and ${ }^{b}$ Departments of Medicine and \\ Epidemiology and Welch Center for Prevention, Epidemiology and Clinical Research, Johns Hopkins University, \\ and Nephrology Center of Maryland, Baltimore, MD, USA
}

In response to the disproportionate burden of hospital readmissions among US end-stage renal disease patients [1], the Centers for Medicare and Medicaid Services (CMS) implemented the standardized readmissions ratio (SRR) as a pay-for-performance measure for hospitals and, recently, for outpatient dialysis facilities. These changes to US policy [2] have created an even more urgent need to find ways to reduce hospital readmissions in the dialysis population. However, according to our knowledge, no US studies to date have explored interventions designed specifically to reduce hospital readmissions among dialysis patients.

In this issue of the American Journal of Nephrology, Wingard et al. [3] report the results of a multi-component, facility-level, pragmatic trial designed to reduce 30 -day readmissions among hemodialysis patients. Overall, 26 intervention and 18 matched control facilities were targeted in the West Virginia/Ohio/Kentucky region. Components of the intervention (collectively, the Right $\mathrm{TraC}^{\mathrm{TM}}$ program) were based on prior work aimed at improving care transitions to reduce hospital readmissions [4] plus the results of a stakeholder root cause analysis. This phased intervention included admission and postdischarge checklists implemented by dialysis facility nurses; a care coordinator assigned to each patient to as-

\section{KARGER}

(c) 2017 S. Karger AG, Basel

E-Mail karger@karger.com

www.karger.com/ajn sist with implementing discharge instructions; a call center support system that allowed for centralized information exchange between providers; and a monthly feedback report for providers throughout all phases. Overall, 30-day readmissions decreased, but there was no statistically significant difference in the pre- to post-intervention change in 30-day readmissions among patients in intervention vs. control facilities. Nonetheless, this work represents an admirable undertaking in which investigators created and implemented a novel intervention in the dialysis facility setting to reduce hospital readmissions in this high-risk population on a regional scale.

The authors acknowledged several limitations. Wingard et al. [3] noted that their results could not be generalized to other dialysis patient populations, particularly to urban dialysis patients who may have differences in terms of demographics, education, and access to care. The authors also noted that, in their pragmatic design, the effects of individual components of the intervention could not be teased apart. However, these are typical features of a

Title of paper upon which editorial is based: "Right TraC'M post-hospitalization care transitions program to reduce readmissions for hemodialysis patients."
Laura C. Plantinga, $\mathrm{PhD}$

Division of Renal Medicine, Department of Medicine Emory University, 101 Woodruff Circle

5105 Woodruff Memorial Building, Atlanta GA-30322 (USA)

E-Mail laura.plantinga@emory.edu 
multicomponent pragmatic trial and can represent the strength of the pragmatic study design: it measures realworld effectiveness of multicomponent interventions that are easily translated to, if not already part of, clinical practice [5].

Indeed, the primary limitation of the study by Wingard et al. [3] was not that the design was pragmatic but that the intervention was not randomized. While facilities were matched on a few facility characteristics and adjustment for patient demographics and select comorbid conditions was performed, some level of residual confounding by facility and patient factors is likely. Other limitations may have led to the observed non-statistically significant results [3]. First, the number of facilities was small and this limited the power to detect the effect of this facility-level intervention. Even if the study had been randomized, facility-level confounding due to chance would have been likely. Second, the analysis accounted for the clustering of admissions among individuals but not of patients within facilities. Third, potential secular trends of declining readmissions and contamination across clinics by hospital and dialysis providers cannot be ruled out. Fourth, fidelity was potentially suboptimal for some components of the intervention: e.g., fewer than half of patients were ever reached by telephone in the 30 days post-discharge by the care coordinator [3]. Finally, some of the intervention components may have been least likely to reach the patients who were most likely to be readmitted. For example, patients who could not be contacted by telephone by the care coordinator/case manager may have had a range of psychosocial issues that decreased consistent telephone access as well as increased readmission risk. Also, a post-discharge checklist had to be completed within 3 dialysis sessions after discharge, but a substantial proportion of readmissions may occur in this early period.

In addition to minimizing these limitations, other issues should be considered carefully in future studies of interventions to reduce readmission in the dialysis population. The inclusion and exclusion criteria for both clinics and patients and the definition of readmissions are critical to the validity of results. One could consider excluding patients who are frequently readmitted, who leave the hospital against medical advice, or who die within 30 days of a hospital discharge, particularly if the goal of the study is to reduce the risk of facility penalties based on the SRR, which does not include such patients [6]. The readmission outcome could be defined as a percentage of index admissions that result in a 30-day readmission; a ratio of observed to expected readmissions (e.g., the SRR [7]); or, as in the study by Wingard et al. [3], a readmis- sion rate per person-year. For the latter, defining time at risk can be complex; patients are technically only at risk for readmission during the 30-day periods following hospital discharges. At this point, none of these approaches is known to be superior, but it will behoove us as a research community to use a limited number of accepted definitions so that future results can be compared across studies. Finally, the approach described by Wingard et al. [3] should be customized for different patient populations. For example, race, age, poverty, geography (urban vs. rural), education, comorbid conditions, and reasons for admission in the target patient population will all greatly affect the choice and design of interventions. The preferences of dialysis facility providers and leadership, such as those elicited in the root cause analysis by Wingard et al. [3], should be considered as well, since interventions without provider buy-in are likely to be incompletely implemented.

It is important to note that, while CMS has instituted the pay-for-performance scheme for hospital readmissions for dialysis patients, it does not provide any payments for infrastructure to allow for increased communication between dialysis providers and hospitals, which is arguably the most critical element in reducing readmissions in dialysis patients. Wingard et al. [3] noted that their intervention components specifically addressing this problem, individual case management, and centralized call support (Dialysis Link ${ }^{\mathrm{TM}}$ ) incurred substantial costs for the dialysis provider. It is unknown whether these costs may be balanced by future cost savings due to reduced readmissions among their patients and subsequent lack of payment penalties related to readmissions. However, to make the initial investment for such interventions may be considerably more difficult for dialysis providers that are not part of a large dialysis organization. Research funding could allow for the testing of such interventions, but the feasibility and sustainability of such interventions will be limited. Strong support from facility leadership and consistent funding for care transitions infrastructure are needed to produce sustainable effects on readmissions [7].

The study by Wingard et al. [3] should inspire researchers to continue to borrow effective interventions from other settings and tailor innovative approaches to reduce readmissions in dialysis patients. Additional pragmatic intervention studies, preferably randomized, are needed, and that too on a large scale. Further, outcomes such as patient mortality and post-discharge emergency department visits and observation stays could be examined along with readmissions. We also need to continue building a solid observational evidence base for dialysis 
readmissions, which remains underdeveloped. Discovering novel correlates of readmissions in dialysis could inform novel approaches to reduce readmissions in this population. Ultimately, reducing readmissions in dialysis patients will not only reduce societal costs but also improve patient-centered attributes of care and quality of life - a win-win-win situation for patients, providers, and policymakers.

\section{Financial Support}

Support for this work was provided by the Agency for Healthcare Research and Quality (R03 HS025018-01).

\section{Disclosure Statement}

None of the authors have any conflicts of interest to report.

\section{References}

1 Jencks SF, Williams MV, Coleman EA: Rehospitalizations among patients in the Medicare fee-for-service program. N Engl J Med 2009;360:1418-1428.

2 Patient Protection and Affordable Care Act, 42 U.S.C. $\$ 18001,2010$.

3 Wingard RL, McDougall K, Axley B, Howard A, O'Keefe C, Armistead N, Lynch JR, Rosen S, Usvyat L, Maddux FW: Right $\mathrm{TraC}^{\mathrm{TM}}$ post-hospitalization care transitions program to reduce readmissions for hemodialysis patients. Am J Nephrol 2017;45:532539.
4 Coleman EA, Parry C, Chalmers S, Min SJ: The care transitions intervention: results of a randomized controlled trial. Arch Intern Med 2006;166:1822-1828.

5 Dember LM, Archdeacon P, Krishnan M, et al: Pragmatic trials in maintenance dialysis: perspectives from the Kidney Health Initiative. J Am Soc Nephrol 2016;27:2955-2963.
6 University of Michigan Kidney Epidemiology and Cost Center: Report for the Standardized Readmission Ratio. Centers for Medicare and Medicaid Services, 2014. https://www.cms. gov/Medicare/Quality-Initiatives-PatientAssessment-Instruments/ESRDQIP/ Downloads/MeasureMethodologyReport fortheProposedSRRMeasure.pdf.

7 Parrish MM, O'Malley K, Adams RI, Adams SR, Coleman EA: Implementation of the care transitions intervention: sustainability and lessons learned. Prof Case Manag 2009;14:282293. 